\title{
Robust-Adaptive Interval Predictive Control for Linear Uncertain Systems
}

\author{
Edouard Leurent and Denis Efimov and Odalric-Ambrym Maillard
}

\begin{abstract}
We consider the problem of stabilization of a linear system, under state and control constraints, and subject to bounded disturbances and unknown parameters in the state matrix. First, using a simple least square solution and available noisy measurements, the set of admissible values for parameters is evaluated. Second, for the estimated set of parameter values and the corresponding linear interval model of the system, two interval predictors are recalled and an unconstrained stabilizing control is designed that uses the predicted intervals. Third, to guarantee the robust constraint satisfaction, a model predictive control algorithm is developed, which is based on solution of an optimization problem posed for the interval predictor. The conditions for recursive feasibility and asymptotic performance are established. Efficiency of the proposed control framework is illustrated by numeric simulations.
\end{abstract}

\section{INTRODUCTION}

There are plenty of real-world control problems for dynamical systems, which face a severe model uncertainty (that can be represented by unknown parameters and exogenous disturbances), under strict state and control constraints, whose maintaining is critical and related with the system safety (e.g., path/trajectory planning for autonomous cars and robots [1], [2]). The most popular approaches aiming to solve these complex regulation issues are the methods based on reinforcement (deep) learning [3] or the Model Predictive Control (MPC) algorithms [4], [5], [6], which are more common in industrial applications (there are also techniques relying on both frameworks as in [2], [7]). The advantages of these tools consist in the ability to provide a robust constraint satisfaction, while ensuring optimization of a selected cost. A shortage is their increased (online) computational complexity, which becomes less important with growing numeric capacities of smart sensors and actuators that are omnipresent nowadays.

Focusing on the latter group of approaches, the adaptive MPC is a common framework to counteract the influence of uncertain parameters [8], [9], [10], [11], [12], which includes, first, an estimation/adaptation algorithm to evaluate the model uncertainty, and second, an MPC algorithm that helps to keep the constraints during transients. If the constraint satisfaction is predominant for system's safety, the complicacy comes from the evaluation of all possible plant trajectories in the presence of external perturbations and noises.

In the present work, our goal is to develop a simple solution that allows the system comportment to be evaluated for given model uncertainty with adjustments provided by estimation

Edouard Leurent is with Renault Group, Paris, France.

Denis Efimov and Odalric-Ambrym Maillard are with Inria Valse and Inria SequeL respectively, Inria Lille Nord-Europe, France. algorithms. Such a solution is based on interval predictors proposed recently [13], [1], whose use will provide to a slightly nonlinear MPC algorithm an ability to keep the constraints in all admissible scenarios.

The outline of this work is as follows. The detailed problem statement is given in Section II We consider a continuous-time linear system with uncertainty presented by state and output disturbances, as well as a vector of unknown parameters belonging to a given compact set; and our goal is the state stabilization while satisfying the state and the control restrictions (due to presence of disturbances the input-to-state stability concept is used). A simple parameter estimation algorithm with evaluation of its accuracy is discussed in III] (this part is rather standard and does not constitute the main novelty). The obtained set of admissible values for the uncertain parameters allows an interval model to be obtained for the considered system. Two interval predictors are introduced in Section IV together with an unconstrained robust stabilizing control that uses the interval predictor variables only. In Section V, an MPC algorithm based on the designed interval predictors is developed, and analyzed in our main result. Applicability of the approach is demonstrated on lane-keeping application for a self-driving car in Section VI

\section{NOTATION}

Denote $[n]=\{1,2, \ldots, n\}$ for any $n \in \mathbb{N}$. Euclidean norm is denoted as $|\cdot|$, and $L_{\infty}$ norm on $\left[t_{0}, t_{1}\right)$ as $\|\cdot\|_{\left[t_{0}, t_{1}\right)}$. We denote as $\mathcal{L}_{\infty}^{m}$ the set of all inputs $u: \mathbb{R}_{+} \rightarrow \mathbb{R}^{m}$ with the property $\|u\|_{[0,+\infty]}<\infty$. Given a matrix $A \in \mathbb{R}^{n \times n}$, denote $A^{+}=\max \{A, 0\}, A^{-}=A^{+}-A,|A|=A^{+}+A^{-}$. For two vectors $x_{1}, x_{2} \in \mathbb{R}^{n}$ or matrices $A_{1}, A_{2} \in \mathbb{R}^{n \times n}$, the relations $x_{1} \leq x_{2}$ and $A_{1} \leq A_{2}$ are understood elementwise. The relation $P \prec 0(P \succeq 0)$ means that a symmetric matrix $P \in \mathbb{R}^{n \times n}$ is negative (positive semi) definite.

\section{Problem Statement}

We consider a linear system:

$$
\dot{x}(t)=A(\theta) x(t)+B u(t)+D \omega(t), t \geq 0,
$$

where $x(t) \in \mathbb{R}^{p}$ is the state, $u(t) \in \mathbb{R}^{q}$ is the control and $\omega(t) \in \mathbb{R}^{r}$ is the state perturbation, $\omega \in \mathcal{L}_{\infty}^{r}$; it is assumed that the constant uncertain parameter vector $\theta \in \mathbb{R}^{d}$ in the state matrix $A: \mathbb{R}^{d} \rightarrow \mathbb{R}^{p \times p}$ belongs to a compact set $\Theta \subset \mathbb{R}^{d}$; the control matrix $B \in \mathbb{R}^{p \times q}$ and disturbance matrix $D \in \mathbb{R}^{p \times r}$ are known. We also assume that the noisy observations of $x(t)$ and and $\dot{x}(t)$ are available:

$$
y_{1}(t)=x(t)+\nu_{1}(t), y_{2}(t)=\dot{x}(t)+\nu_{2}(t),
$$


where $\nu(t)=\left[\nu_{1}^{\top}(t) \nu_{2}^{\top}(t)\right]^{\top} \in \mathbb{R}^{2 p}$ is the measurement disturbance, $\nu \in \mathcal{L}_{\infty}^{2 p}$. Roughly speaking, we assume with (2) that the state $x(t)$ and its derivative $\dot{x}(t)$ are estimated using some observation/differentiation approaches [14], [15].

Assumption 1. There exist signals $\underline{\omega}, \bar{\omega} \in \mathcal{L}_{\infty}^{r}, \underline{\nu}, \bar{\nu} \in \mathcal{L}_{\infty}^{2 p}$ and two vectors $\underline{x}_{0}, \bar{x}_{0} \in \mathbb{R}^{p}$ such that

$$
\begin{gathered}
\underline{\omega}(t) \leq \omega(t) \leq \bar{\omega}(t), \underline{\nu}(t) \leq \nu(t) \leq \bar{\nu}(t) \quad \forall t \geq 0 \\
\underline{x}_{0} \leq x(0) \leq \bar{x}_{0} .
\end{gathered}
$$

\section{A. Problem}

Our goal is to design a robust control that stabilizes (1), (2) at a vicinity of the origin under Assumption 1 such that

$$
x(t) \in \mathbb{X}, u(t) \in \mathbb{U} \quad \forall t \geq 0,
$$

where $\left[\underline{x}_{0}, \bar{x}_{0}\right] \subset \mathbb{X} \subset \mathbb{R}^{p}$ and $\mathbb{U} \subset \mathbb{R}^{q}$ are given bounded constraint sets for the state and the control, respectively.

\section{MOdEl Estimation}

To derive a confidence region $\hat{\Theta}(t) \subseteq \Theta$ for the uncertain parameters $\theta$, the structure of $A(\theta)$ must be specified:

Assumption 2. There exist known matrices $A, \phi_{1}, \ldots, \phi_{d} \in$ $\mathbb{R}^{p \times p}$ such that for all $\theta \in \Theta$,

$$
A(\theta)=A+\sum_{i=1}^{d} \theta_{i} \phi_{i}
$$

(1), (2) and Assumption 2 yield the linear regression:

$$
y(t)=\Phi(t) \theta+\eta(t),
$$

where $y(t)=y_{2}(t)-A y_{1}(t)-B u(t)$ and $\Phi(t)=$ $\left[\phi_{1} y_{1}(t) \ldots \phi_{d} y_{1}(t)\right] \in \mathbb{R}^{p \times d}$ are known signals, and

$$
\eta(t)=D \omega(t)-\left(A+\sum_{i=1}^{d} \theta_{i} \phi_{i}\right) \nu_{1}(t)+\nu_{2}(t)
$$

is the combined perturbation, which is from $\mathcal{L}_{\infty}^{p}$ under assumptions 1 and 2 . $\|\eta\|_{\infty} \leq \bar{\eta}$.

We need a hypothesis on the level of excitation of $\Phi$ [16]:

Assumption 3. There exist $\ell>0$ and $\vartheta>0$ such that the matrix function $\Phi: \mathbb{R}_{+} \rightarrow \mathbb{R}^{p \times d}$ satisfies $(\ell, \vartheta)$-Persistence of Excitation (PE) condition: for any $t \in \mathbb{R}_{+}$,

$$
\int_{t}^{t+\ell} \Phi^{\top}(s) \Phi(s) d s \geq \vartheta I_{d} .
$$

Under Assumption 3 for any $g \geq 1$ and $g \ell<T \leq(g+1) \ell$,

$$
\begin{aligned}
\int_{t}^{t+T} \Phi^{\top} \Phi(s) d s & =\left(\int_{t+g \ell}^{t+T}+\sum_{i=0}^{g-1} \int_{t+i \ell}^{t+(i+1) \ell}\right) \Phi^{\top} \Phi(s) d s \\
& \geq \frac{g}{g+1} \frac{\vartheta}{\ell} T I_{d} \geq \frac{\vartheta}{2 \ell} T I_{d},
\end{aligned}
$$

i.e., the matrix function $\int_{t}^{t+T} \Phi^{\top}(s) \Phi(s) d s$ is nonsingular. Taking into account this observation, in order to solve (4) and obtain $\hat{\Theta}(t)$, we use next the simplest least square estimation:

$$
\hat{\theta}(t)=\left\{\begin{array}{ll}
\left(\int_{0}^{t} \Phi^{\top}(s) \Phi(s) d s\right)^{-1} \int_{0}^{t} \Phi^{\top}(s) y(s) d s & t \geq \ell \\
\theta_{0} & t \in[0, \ell)
\end{array},\right.
$$

where $\hat{\theta}(t) \in \mathbb{R}^{d}$ is an estimate of $\theta$ and $\theta_{0} \in \Theta$ is an initial estimate. The estimation error of (5) can be evaluated as:

Proposition 1. Let assumptions 1] 2, and 3 be satisfied. Then, for all $t \geq \ell:|\hat{\theta}(t)-\theta| \leq \Delta \theta\left(\|x\|_{\infty}\right)$, where

$\Delta \theta\left(\|x\|_{\infty}\right)=\frac{2 \ell}{\vartheta} \max _{i \in[d]}\left\|\phi_{i}\right\|_{2}\left(\|x\|_{\infty}+\max \left\{\left\|\underline{\nu}_{1}\right\|_{\infty},\left\|\bar{\nu}_{1}\right\|_{\infty}\right\}\right) \bar{\eta}$.

Proof. According to (4), the algorithm (5) can be rewritten for $t \geq \ell$ in the form:

$$
\hat{\theta}(t)=\theta+\left(\int_{0}^{t} \Phi^{\top}(s) \Phi(s) d s\right)^{-1} \int_{0}^{t} \Phi^{\top}(s) \eta(s) d s .
$$

Using the fact that $\int_{0}^{t} \Phi^{\top}(s) \Phi(s) d s \geq \frac{\vartheta}{2 \ell} t I_{d}$ for $t \geq \ell$, the claim follows the direct computations:

$$
\begin{aligned}
|\hat{\theta}(t)-\theta| & \leq\left\|\left(\int_{0}^{t} \Phi^{\top}(s) \Phi(s) d s\right)^{-1}\right\|\left|\int_{2}^{t} \Phi^{\top}(s) \eta(s) d s\right| \\
& \leq \frac{2 \ell}{\vartheta t} \int_{0}^{t}\|\Phi(s)\|_{2}|\eta(s)| d s \leq \text { claimed bound. }
\end{aligned}
$$

To guarantee the robust constraint satisfaction we have to take into account not only an estimate $\hat{\theta}(t)$ of the vector of uncertain parameters $\theta$, but the set of all admissible values $\hat{\Theta}(t) \subseteq \Theta$ (with $\theta, \hat{\theta}(t) \in \hat{\Theta}(t)$ for all $t \geq 0$ ). Following the result of Proposition 1 we can calculate an estimate for $\hat{\Theta}(t)$ :

$$
\hat{\Theta}(t)=\Theta \bigcap_{\tau \in[\ell, t]}\left\{\widetilde{\theta} \in \mathbb{R}^{d}:|\hat{\theta}(\tau)-\widetilde{\theta}| \leq \Delta \theta(X)\right\} .
$$

The property $\hat{\Theta}(t) \subseteq \Theta$ is satisfied for all $t \geq 0$, and the size of $\hat{\Theta}(t)$ is shrinking. We also can use some updated estimates on $\|x\|_{\infty}$ in (6) instead of the worst case bound $X$.

\section{State Prediction}

We aim to derive an interval predictor [17], [1] for the system (1), which takes the information on the observed current state $x(t) \in\left[y_{1}(t)-\underline{\nu}_{1}(t), y_{1}(t)+\bar{\nu}_{1}(t)\right]$, the estimated confidence region $\hat{\Theta}(t)$, a planned control signal $u:[t,+\infty) \rightarrow \mathbb{R}^{q}$ and the admissible bounds on the state perturbation $[\underline{\omega}(t), \bar{\omega}(t)]$; and outputs an interval $[\underline{x}(t), \bar{x}(t)]$ that must verify the inclusion property:

$$
\underline{x}(s) \leq x(s) \leq \bar{x}(s), \quad \forall s \geq t .
$$

There exist many predictors based, e.g., on zonotope [18] or interval [17], [1] representation of the set of admissible values of $x(s)$. Opting the simplicity of implementation and computational efficiency, we use an interval predictor here that ensures the property (7). To this end we will assume that the set $\hat{\Theta}(t)$ computed by 6 is given, then there are two possible representations of uncertainty of $A(\theta)$ in (1):

- interval: for all $\theta \in \hat{\Theta}(t)$ and some $\underline{A}, \bar{A} \in \mathbb{R}^{p \times p}$,

$$
\underline{A} \leq A(\theta) \leq \bar{A}
$$


- polytopic: for all $\theta \in \hat{\Theta}(t)$ and some $A_{0}=A(\hat{\theta}(t))$ and $\Delta A_{i}=h_{i} \Delta \theta(X)$ for $h_{i} \in\{-1,1\}^{d}$ with $i \in[2 d]$,

$$
A(\theta) \in\left\{A_{0}+\sum_{i=1}^{2^{d}} \alpha_{i} \Delta A_{i}: \alpha_{i} \geq 0, \sum_{i=1}^{2^{d}} \alpha_{i}=1\right\}
$$

The matrices $\underline{A}, \bar{A}$ can be calculated using the interval arithmetic for $\hat{\Theta}(t)$; suggestions for selection of $A_{0}$ and $\Delta A_{i}$ are given in 9] (other variants can be used [19]).

\section{A. Design of predictors}

A simple solution providing (7) is proposed in [13], where the matrix interval arithmetic is used to derive the predictor:

Proposition 2 (Simple predictor of [13]). Assume that Assumption 1 and the relations (8) are satisfied for the system (11). Then for $s \geq t$ the interval predictor,

$$
\begin{aligned}
\underline{\dot{x}}(s)= & \underline{A}^{+} \underline{x}^{+}(s)-\bar{A}^{+} \underline{x}^{-}(s)-\underline{A}^{-} \bar{x}^{+}(s)+\bar{A}^{-} \bar{x}^{-}(s) \\
& +B u(s)+D^{+} \underline{\omega}(s)-D^{-} \bar{\omega}(s), \\
\dot{\bar{x}}(s)= & \bar{A}^{+} \bar{x}^{+}(s)-\underline{A}^{+} \bar{x}^{-}(s)-\bar{A} \underline{x}^{+}(s)+\underline{A}^{-} \underline{x}^{-}(s) \\
& +B u(s)+D^{+} \bar{\omega}(s)-D^{-} \underline{\omega}(s), \\
& \underline{x}(t)=y_{1}(t)-\underline{\nu}_{1}(t), \bar{x}(t)=y_{1}(t)+\bar{\nu}_{1}(t),
\end{aligned}
$$

ensures the inclusion property (7).

However, [1] showed that this predictor can have unstable dynamics, even for stable systems, which causes a fast explosion of the interval width $\bar{x}(s)-\underline{x}(s)$. In that work, an enhanced predictor is proposed, which exploits the polytopic structure (9) to produce tighter and more stable predictions, at the price of an additional requirement:

Assumption 4. There exists a nonsingular matrix $Z \in \mathbb{R}^{p \times p}$ such that $Z^{-1} A_{0} Z$ is Metzler

In practice, this assumption is often verified. It is for instance the case whenever $A_{0}$ is diagonalizable, or a method from [20] computes a similarity transformation $Z$ when the system is observable with respect to a scalar output. To simplify the notation, we further assume that $Z=I_{p}$. Denote $\Delta A_{+}=\sum_{i=1}^{2^{d}} \Delta A_{i}^{+}$and $\Delta A_{-}=\sum_{i=1}^{2^{d}} \Delta A_{i}^{-}$.

Proposition 3 (Enhanced predictor of [1]). Assume that assumptions 14 and the relation (9) are satisfied for the system (1). Then for $s \geq t$ the interval predictor,

$$
\begin{aligned}
\underline{\dot{x}}(s)= & A_{0} \underline{x}(s)-\Delta A_{+} \underline{x}^{-}(s)-\Delta A_{-} \bar{x}^{+}(s) \\
& +B u(s)+D^{+} \underline{\omega}(s)-D^{-} \bar{\omega}(s), \\
\dot{\bar{x}}(s)= & A_{0} \bar{x}(s)+\Delta A_{+} \bar{x}^{+}(s)+\Delta A_{-} \underline{x}^{-}(s) \\
& +B u(s)+D^{+} \bar{\omega}(s)-D^{-} \underline{\omega}(s), \\
& \underline{x}(t)=y_{1}(t)-\underline{\nu}_{1}(t), \bar{x}(t)=y_{1}(t)+\bar{\nu}_{1}(t),
\end{aligned}
$$

ensures the inclusion property (7).

In Fig. 1. the difference in stability of two predictors (10) and (11) is illustrated for a simple example. [1] suggest to

\footnotetext{
${ }^{1}$ We say that a matrix is Metzler when all its non-diagonal coefficients are non-negative.
}

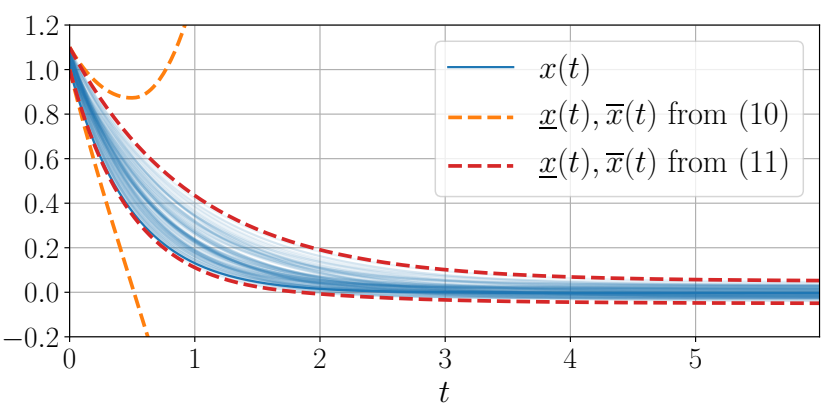

Fig. 1. Comparison of 10 and 11 for a simple system $\dot{x}(t)=-\theta x(t)+$ $\omega(t)$, with $\theta \in[1,2]$ and $\omega(t) \in[-0.05,0.05]$.

always prefer (11) whenever Assumption 4 is verified, and only fallback to 10$]$ as a last resort.

\section{B. Stabilizing control for (10) and (11)}

Note that both interval predictors, (10) and (11), admit a representation in the form (we use the time argument $t=s$ in this subsection):

$$
\dot{\xi}(t)=\mathcal{A}_{0} \xi(t)+\mathcal{A}_{1} \xi^{+}(t)+\mathcal{A}_{2} \xi^{-}(t)+\mathcal{B} u(t)+\delta(t),
$$

where $\xi(t)=\left[\underline{x}^{\top}(t) \bar{x}^{\top}(t)\right]^{\top} \in \mathbb{R}^{2 p}$ is the extended state vector of the predictors,

$$
\delta(t)=\left[\begin{array}{cc}
D^{+} & -D^{-} \\
-D^{-} & D^{+}
\end{array}\right]\left[\begin{array}{c}
\underline{\omega}(t) \\
\bar{\omega}(t)
\end{array}\right] \in \mathbb{R}^{2 p}
$$

is the external known input, $\mathcal{B}=\left[B^{\top} B^{\top}\right]^{\top}$,

$\mathcal{A}_{0}=0, \mathcal{A}_{1}=\left[\begin{array}{cc}\underline{A}^{+} & -\underline{A}^{-} \\ -\bar{A}^{-} & \bar{A}^{+}\end{array}\right], \mathcal{A}_{2}=\left[\begin{array}{cc}-\bar{A}^{+} & \bar{A}^{-} \\ \underline{A}^{-} & -\underline{A}^{+}\end{array}\right]$

for 10$)$ and

$\mathcal{A}_{0}=\left[\begin{array}{cc}A_{0} & 0 \\ 0 & A_{0}\end{array}\right], \mathcal{A}_{1}=\left[\begin{array}{cc}0 & -\Delta A_{-} \\ 0 & \Delta A_{+}\end{array}\right], \mathcal{A}_{2}=\left[\begin{array}{cc}-\Delta A_{+} & 0 \\ \Delta A_{-} & 0\end{array}\right]$

for (11). Note that (12) is a nonlinear system due to the presence of globally Lipschitz nonlinearities $\xi^{+}(t)$ and $\xi^{-}(t)$.

Due to (7), the boundedness of $\xi(t)$ implies the same property of $x(t)$. Therefore, in order to regulate (1) it is required to design a state feedback $u(t)$ minimizing the asymptotic amplitude of the state $\xi(t)$ for given input $\delta(t)$ [21]. In other words, it is necessary to design a control $u(t)$ that input-to-state stabilizes (12). It is proposed to look for such a control in the form:

$$
u(t)=K_{0} \xi(t)+K_{1} \xi^{+}(t)+K_{2} \xi^{-}(t)+S \delta(t),
$$

where $K_{0}, K_{1}, K_{2} \in \mathbb{R}^{q \times 2 p}$ and $S \in \mathbb{R}^{q \times 2 p}$ are the gains to be designed (13) contains a nonlinear feedback). The selection of $S$ is simple, it has to minimize the norm of $\mathcal{B} S+I_{2 p}$, and it can be made independently of $K_{0}, K_{1}, K_{2}$. Therefore, denoting $\widetilde{\delta}(t)=\left(\mathcal{B} S+I_{2 p}\right) \delta(t)$ the closed-loop system (12), 13) takes the form:

$$
\dot{\xi}(t)=\mathcal{D}_{0} \xi(t)+\mathcal{D}_{1} \xi^{+}(t)+\mathcal{D}_{2} \xi^{-}(t)+\widetilde{\delta}(t),
$$

where $\mathcal{D}_{i}=\mathcal{A}_{i}+\mathcal{B} K_{i}$ for $i \in[3]$, and the restrictions, which the gains $K_{0}, K_{1}, K_{2}$ have to respect, are given below: 
Theorem 1. If there exist diagonal matrices $P, Q, Q_{+}, Q_{-}$, $Z_{+}, Z_{-}, \Psi_{+}, \Psi_{-}, \Psi, \Gamma \in \mathbb{R}^{2 p \times 2 p}$ such that the following linear matrix inequalities are satisfied:

$$
\begin{gathered}
P+\min \left\{Z_{+}, Z_{-}\right\}>0, \Upsilon \preceq 0, \Gamma>0, \\
Q+\min \left\{Q_{+}, Q_{-}\right\}+2 \min \left\{\Psi_{+}, \Psi_{-}\right\}>0 \\
\text { where } \Upsilon=\left[\begin{array}{cccc}
\Upsilon_{11} & \Upsilon_{12} & \Upsilon_{13} & P \\
\Upsilon_{12}^{\top} & \Upsilon_{22} & \Upsilon_{23} & Z_{+} \\
\Upsilon_{13}^{\top} & \Upsilon_{23}^{\top} & \Upsilon_{33} & -Z_{-} \\
P & Z_{+} & -Z_{-} & -\Gamma
\end{array}\right] \\
\Upsilon_{11}=\mathcal{D}_{0}^{\top} P+P \mathcal{D}_{0}+Q, \Upsilon_{12}=\mathcal{D}_{0}^{\top} Z_{+}+P \mathcal{D}_{1}+\Psi_{+}, \\
\Upsilon_{13}=P \mathcal{D}_{2}-\mathcal{D}_{0}^{\top} Z_{-}-\Psi, \Upsilon_{22}=Z_{+} \mathcal{D}_{1}+\mathcal{D}_{1}^{\top} Z_{+}+Q_{+}, \\
\Upsilon_{23}=Z_{+} \mathcal{D}_{2}-\mathcal{D}_{1}^{\top} Z_{-}+\Psi, \Upsilon_{33}=-Z_{-} \mathcal{D}_{2}-\mathcal{D}_{2}^{\top} Z_{-}+Q_{-},
\end{gathered}
$$

then (14) is input-to-state stable with respect to $\underline{\omega}, \bar{\omega}$.

Note that the requirement that $P$ has to be diagonal is not restrictive, since for a Metzler matrix $\mathcal{D}_{0}$ (the case of 110$)$ and (11), its stability is equivalent to existence of a diagonal solution $P$ of the Lyapunov equation $\mathcal{D}_{0}^{\top} P+P \mathcal{D}_{0} \prec 0$ [22].

Proof. Consider a candidate Lyapunov function:

$$
\begin{gathered}
V(\xi)=\xi^{\top} P \xi+\xi^{\top} Z_{+} \xi^{+}-\xi^{\top} Z_{-} \xi^{-} \\
=\sum_{k=1}^{2 p} P_{k, k} \xi_{k}^{2}+\left(Z_{+}\right)_{k, k}\left|\xi_{k}\right| \xi_{k}^{+}+\left(Z_{-}\right)_{k, k}\left|\xi_{k}\right| \xi_{k}^{-},
\end{gathered}
$$

which is positive definite provided that $P+\min \left\{Z_{+}, Z_{-}\right\}>0$ since all terms in $V$ are quadratic-like, and whose derivative for the system (14) dynamics takes the form:

$$
\begin{gathered}
\dot{V}=2 \dot{\xi}^{\top} P \xi+2 \dot{\xi}^{\top} Z_{+} \xi^{+}-2 \dot{\xi}^{\top} Z_{-} \xi^{-} \\
=\left[\begin{array}{c}
\xi \\
\xi^{+} \\
\xi^{-} \\
\widetilde{\delta}
\end{array}\right]^{\top} \Upsilon\left[\begin{array}{c}
\xi \\
\xi^{+} \\
\xi^{-} \\
\widetilde{\delta}
\end{array}\right]-\xi^{\top} Q \xi-\left(\xi^{+}\right)^{\top} Q_{+} \xi^{+} \\
-\left(\xi^{-}\right)^{\top} Q_{-} \xi^{-}-2\left(\xi^{+}\right)^{\top} \Psi \xi^{-}-2\left(\xi^{+}\right)^{\top} \Psi_{+} \xi \\
-2\left(-\xi^{-}\right)^{\top} \Psi_{-} \xi+\widetilde{\delta}^{\top} \Gamma \widetilde{\delta} .
\end{gathered}
$$

Note that $\left(\xi^{+}\right)^{\top} \Psi \xi^{-}=0,\left(\xi^{+}\right)^{\top} \Psi_{+} \xi \geq 0,\left(-\xi^{-}\right)^{\top} \Psi_{-} \xi \geq$ 0 for any diagonal matrix $\Psi$ and $\Psi_{+} \geq 0, \Psi_{-} \geq 0$. Hence, if $\Upsilon \preceq 0$, as it is assumed in the theorem, we obtain that

$$
\begin{aligned}
\dot{V} \leq & -\xi^{\top} Q \xi-\left(\xi^{+}\right)^{\top} Q_{+} \xi^{+}-\left(\xi^{-}\right)^{\top} Q_{-} \xi^{-} \\
& -2\left(\xi^{+}\right)^{\top} \Psi_{+} \xi-2\left(-\xi^{-}\right)^{\top} \Psi_{-} \xi+\widetilde{\delta}^{\top} \Gamma \tilde{\delta} \\
\leq & -\xi^{\top} \Omega \xi+\widetilde{\delta}^{\top} \Gamma \widetilde{\delta},
\end{aligned}
$$

where $\Omega=Q+\min \left\{Q_{+}, Q_{-}\right\}+2 \min \left\{\Psi_{+}, \Psi_{-}\right\}>0$ is a diagonal matrix. The substantiated properties of $V$ and its derivative imply that (14) is input-to-state stable [23], [24] with respect to the input $\delta$ (or, by its definition, to $(\underline{\omega}, \bar{\omega})$ ).

Following the proof of Theorem 1 , for all $\xi \in \mathbb{R}^{2 p}$,

$$
\xi^{\top}\left(P+\min \left\{Z_{+}, Z_{-}\right\}\right) \xi \leq V(\xi) \leq \xi^{\top}\left(P+Z_{+}^{+}+Z_{-}^{+}\right) \xi,
$$

then $\dot{V} \leq-\alpha V+\widetilde{\delta}^{\top} \Gamma \widetilde{\delta}$ for all $\xi, \widetilde{\delta} \in \mathbb{R}^{2 p}$, where $\alpha=$ $\min _{i \in[2 p]} \lambda_{i}\left(\Omega\left(P+Z_{+}^{+}+Z_{-}^{+}\right)^{-1}\right)$, and we can define the set (recall that the signal $\widetilde{\delta}(t)$ is known for all $t \geq 0$ )

$$
\mathbb{X}_{f}=\left\{\xi \in \mathbb{R}^{2 p}: V(\xi) \leq \alpha^{-1} \sup _{t \geq 0}\left|\widetilde{\delta}^{\top}(t) \Gamma \widetilde{\delta}(t)\right|\right\},
$$

as the set that asymptotically attracts all trajectories in (14).

The conditions of Theorem 1 assume that the control gains $K_{0}, K_{1}, K_{2}$ are given, let us find these gains as solutions of linear matrix inequalities:

Corollary 1. If there exist diagonal matrices $P, \widetilde{Q}, \widetilde{Q}_{+}, \widetilde{Q}_{-}$, $Z_{+}, Z_{-}, \widetilde{\Psi}_{+}, \widetilde{\Psi}_{-}, \widetilde{\Psi}, \Gamma \in \mathbb{R}^{2 p \times 2 p}$ and matrices $U_{0}, U_{1}, U_{2} \in$ $\mathbb{R}^{q \times 2 p}$ satisfying following linear matrix inequalities:

$$
\begin{gathered}
P>0, Z_{+}>0, Z_{-}>0, \Pi \preceq 0, \Gamma>0, \\
\widetilde{Q}+\min \left\{\widetilde{Q}_{+}, \widetilde{Q}_{-}\right\}+\min \left\{\widetilde{\Psi}_{+}, \widetilde{\Psi}_{-}\right\}=0, \\
\text { where } \quad \Pi=\left[\begin{array}{cccc}
\Pi_{11} & \Pi_{12} & \Pi_{13} & I \\
\Pi_{12}^{\top} & \Pi_{22} & \Pi_{23} & I \\
\Pi_{13}^{\top} & \Pi_{23}^{\top} & \Pi_{33} & -I \\
I & I & -I & -\Gamma
\end{array}\right], \\
\Pi_{11}=P^{-1} \mathcal{A}_{0}^{\top}+\mathcal{A}_{0} P^{-1}+U_{0}^{\top} \mathcal{B}^{\top}+\mathcal{B} U_{0}+\widetilde{Q}, \\
\Pi_{12}=\mathcal{A}_{1} Z_{+}^{-1}+\mathcal{B} U_{1}+P^{-1} \mathcal{A}_{0}^{\top}+U_{0}^{\top} \mathcal{B}^{\top}+\widetilde{\Psi}_{+}, \\
\Pi_{13}=\mathcal{A}_{2} Z_{-}^{-1}+\mathcal{B} U_{2}-P^{-1} \mathcal{A}_{0}^{\top}-U_{0}^{\top} \mathcal{B}^{\top}-\widetilde{\Psi}_{-}, \\
\Pi_{22}=Z_{+}^{-1} \mathcal{A}_{1}^{\top}+\mathcal{A}_{1} Z_{+}^{-1}+U_{1}^{\top} \mathcal{B}^{\top}+\mathcal{B} U_{1}+\widetilde{Q}_{+}, \\
\Pi_{23}=\mathcal{A}_{2} Z_{-}^{-1}+\mathcal{B} U_{2}-Z_{+}^{-1} \mathcal{A}_{1}^{\top}-U_{1}^{\top} \mathcal{B}^{\top}+\widetilde{\Psi}^{-1}, \\
\Pi_{33}=\widetilde{Q}_{-}-Z_{-}^{-1} \mathcal{A}_{2}^{\top}-\mathcal{A}_{2} Z_{-}^{-1}-U_{2}^{\top} \mathcal{B}^{\top}-\mathcal{B} U_{2},
\end{gathered}
$$

then (14) for $K_{0}=U_{0} P, K_{1}=U_{1} Z_{+}$and $K_{2}=U_{2} Z_{-}$is input-to-state stable with respect to the inputs $\underline{\omega}, \bar{\omega}$.

Proof. Note that the conditions $P>0, Z_{+}>0, Z_{-}>0$ imply $P+\min \left\{Z_{+}, Z_{-}\right\}>0$, and

$$
\Upsilon=\left[\begin{array}{cccc}
P & 0 & 0 & 0 \\
0 & Z_{+} & 0 & 0 \\
0 & 0 & Z_{-} & 0 \\
0 & 0 & 0 & I_{2 p}
\end{array}\right] \Pi\left[\begin{array}{cccc}
P & 0 & 0 & 0 \\
0 & Z_{+} & 0 & 0 \\
0 & 0 & Z_{-} & 0 \\
0 & 0 & 0 & I_{2 p}
\end{array}\right]
$$

under substitution $U_{0}=K_{0} P^{-1}, U_{1}=K_{1} Z_{+}^{-1}, U_{2}=$ $K_{2} Z_{-}^{-1}, \widetilde{Q}=P^{-1} Q P^{-1}, \widetilde{Q}_{+}=Z_{+}^{-1} Q_{+} Z_{+}^{-1}, \widetilde{Q}_{-}=$ $Z_{-}^{-1} Q_{-} Z_{-}^{-1}, \widetilde{\Psi}=Z_{-}^{-1} \Psi Z_{+}^{-1}, \widetilde{\Psi}_{+}=P^{-1} \Psi_{+} Z_{+}^{-1}$ and $\widetilde{\Psi}_{-}=P^{-1} \Psi_{-} Z_{-}^{-1}$. Hence, $\Upsilon \preceq 0$ provided that $\Pi \preceq 0$. The inequalities $\widetilde{Q}+\min \left\{\widetilde{Q}_{+}, \widetilde{Q}_{-}\right\}+2 \min \left\{\widetilde{\Psi}_{+}, \widetilde{\Psi}_{-}\right\}>0$ and $Q+\min \left\{Q_{+}, Q_{-}\right\}+2 \min \left\{\Psi_{+}, \Psi_{-}\right\}>0$ are equivalent due to the diagonal structure of all matrices. Therefore, under introduced restrictions all conditions of Theorem 1 are verified for $K_{0}=U_{0} P, K_{1}=U_{1} Z_{+}$and $K_{2}=U_{2} Z_{-}$.

The requirements imposed on $P, Z_{+}, Z_{-}$in this corollary are more restrictive than the conditions of Theorem 1 , but it allows the gains $K_{0}, K_{1}, K_{2}$ to be efficiently calculated.

Under conditions of Theorem 11, the control (13) ensures stabilization of the predictor (12) in a vicinity $\mathbb{X}_{f}$ of the origin whose size is proportional to the system (1) uncertainty (it can be optimized by the choice of $K_{0}, K_{1}, K_{2}$ ). Due to (7), the system (11) also will reach a neighborhood of the origin and the posed control problem would be solved provided that (3) holds. In order to ensure the robust constraint satisfaction we consider an MPC design in the next section. 


\section{Robust CONTROL}

For brevity, the results of this section are given for the predictor 11 only. We need the following hypothesis:

Assumption 5. There exist $K_{0}, K_{1}, K_{2} \in \mathbb{R}^{q \times 2 p}$ satisfying the conditions of Theorem 1 for the matrices $A_{0}$ and $\Delta A_{i}$ with $i \in[2 d]$ calculated in (9) for $\hat{\Theta}(t)=\Theta$, and $\mathbb{X}_{f} \subset \mathbb{X}^{2}$, where the corresponding set $\mathbb{X}_{f}$ is given in 115 , and

$$
K_{0} \xi+K_{1} \xi^{+}+K_{2} \xi^{-}+S \delta(t) \in \mathbb{U}
$$

for any $\xi \in \mathbb{X}_{f}$ and $t \geq 0$.

These properties guarantee that there exists a control (13) that can be always applied to stabilize the predictor (11) and into the set $\mathbb{X}_{f}$ the restrictions (3) also hold for such a control. Define $T>0$ and $\tau \in(0, T)$ as the interval of prediction and the application time for MPC. Denote $t_{i}=i \tau$ for $i \in \mathbb{N}_{+}$, then the developed MPC algorithm can be formalized as:

1) Take $\hat{\Theta}\left(t_{i}\right)$ from 6 and calculate the matrices $A_{0}$ and $\Delta A_{i}$ with $i \in[2 d]$ for (9]).

2) Find, given weights $W_{i} \succeq 0$ in $\mathbb{R}^{2 p \times 2 p}$, the controls

$$
\begin{gathered}
\mathcal{U}=\operatorname{argmin}_{u:\left[t_{i}, t_{i}+T\right] \rightarrow \mathbb{R}^{q}} \xi^{\top}\left(t_{i}+T\right) W_{1} \xi\left(t_{i}+T\right) \\
+\int_{t_{i}}^{t_{i}+T} \xi^{\top}(s) W_{2} \xi(s)+u^{\top}(s) W_{3} u(s) d s
\end{gathered}
$$

such that the following constraints are satisfied:

a) $\xi:\left[t_{i}, t_{i}+T\right] \rightarrow \mathbb{R}^{2 p}$ is a solution of (11)

b) $\xi(s) \in \mathbb{X}^{2}$ and $u(s) \in \mathbb{U}$ for $s \in\left[t_{i}, t_{i}+T\right]$;

c) $\xi\left(t_{i}+T\right) \in \mathbb{X}_{f}$.

3) For $t \in\left[t_{i}, t_{i}+\tau\right)$ select

$$
u(t)= \begin{cases}\mathcal{U}(t) & \xi\left(t_{i}\right) \notin \mathbb{X}_{f} \\ 13 & \xi\left(t_{i}\right) \in \mathbb{X}_{f}\end{cases}
$$

where $K_{0}, K_{1}, K_{2}$ are taken from Assumption 5

As we can conclude, the idea of the proposed dual MPC scheme (see also [25], [5], [6]) is to use an open-loop optimal control to reach a neighborhood of the origin $\mathbb{X}_{f}$ ensuring a robust constraint satisfaction (3), where a closed-loop control (13) can be applied, which provides asymptotic performances (stability and robustness, also with constraint satisfaction due to Assumption 5 and the definition of the terminal set (15)).

The main result of the paper is as follows:

Theorem 2. Let $\underline{x}_{0}, \bar{x}_{0} \in \mathbb{X}$, and assumptions 175 hold with $\bar{\omega}, \bar{\omega}-\underline{\omega}$ being non-increasing functions of $t \geq 0$. Then the closed-loop system given by (1), 2,, 11) and (17) has the following properties:

1) Input-to-state stability for $\underline{x}, \bar{x}$ and practical input-tostate stability for $x$ with respect to $\underline{\omega}, \bar{\omega}$ in the terminal set $\mathbb{X}_{f}$;

2) Recursive feasibility with reaching $\mathbb{X}_{f}$ in a finite time;

3) Constraint satisfaction.

Proof. Recall that $\theta \in \hat{\Theta}(t)$ for all $t \geq 0$ due to the result of Proposition 1, and the size of the set $\hat{\Theta}(t)$ is not growing with time by definition of (6).
1) Note that if for some $t_{k} \geq 0$ the initial conditions $\left(\underline{x}^{\top}\left(t_{k}\right), \bar{x}^{\top}\left(t_{k}\right)\right)^{\top} \in \mathbb{X}_{f} \subset \mathbb{X}^{2}$, then the control (17) equals to (13). According to the definition (15) of $\mathbb{X}_{f}$ and Assumption 5. $\xi(t) \in \mathbb{X}_{f}$ and $u(t) \in \mathbb{U}$ for all $t \geq t_{k}$, and the system is input-to-state stable with respect to $\xi(t)=\left[\underline{x}^{\top}(t) \bar{x}^{\top}(t)\right]^{\top}$ due to the result of Theorem 1 Since $|x(t)| \leq|\xi(t)|$ under (7) for $t \geq t_{k}$ and $\left|\xi\left(t_{k}\right)\right| \leq\left|x\left(t_{k}\right)\right|+\zeta$ with $\zeta>0$, the practical input-to-state stability for the variable

2) Now, let $\left(\underline{x}^{\top}(0), \bar{x}^{\top}(0)\right)^{\top} \in \mathbb{X}^{2} \backslash \mathbb{X}_{f}$ and assume that there is a solution of the optimal control problem (16). Applying such a control through (17) for $t \in[0, \tau)$, we have that $\xi(t) \in \mathbb{X}$ and $u(t) \in \mathbb{U}$ on this time interval. At $t=t_{1}=$ $\tau$, if again $\left(\underline{x}^{\top}\left(t_{1}\right), \bar{x}^{\top}\left(t_{1}\right)\right)^{\top} \in \mathbb{X}^{2} \backslash \mathbb{X}_{f}$, then it recursively exists a solution to 16 since the set $\hat{\Theta}(t)$ is shrinking by its design and the signals $\bar{\omega}(t), \bar{\omega}(t)-\underline{\omega}(t)$ are non-increasing by hypotheses of the theorem (i.e., the solution obtained at $t_{i}$ is a sub-optimal branch of the solution calculated at $t_{i-1}$ for all $i \geq 1$ ). Thus, recursive feasibility follows. Note that $\mathbb{X}_{f}$ is a neighborhood of the origin, and the given in 16 cost with positive definite matrices $W_{1}, W_{2}$ and $W_{3}$ is minimized inside $\mathbb{X}_{f}$. Using this and sub-optimality arguments, since $\xi\left(t_{i}+T\right) \in \mathbb{X}_{f}$ in $[16$ (provided that the optimal control $\mathcal{U}$ is applied) and $\left[\underline{x}\left(t_{i}\right), \bar{x}\left(t_{i}\right)\right] \subset\left[\underline{x}\left(t_{i-1}+\tau\right), \bar{x}\left(t_{i-1}+\tau\right)\right]$ for all $i \geq 1$, there is a finite time instant $t_{k} \geq T$ such that $\left(\underline{x}^{\top}\left(t_{k}\right), \bar{x}^{\top}\left(t_{k}\right)\right)^{\top} \in \mathbb{X}_{f}$, and the system further stays there.

$3)$ is a consequence of the previous analysis: under the control (17) the constrains (3) are always satisfied.

\section{NUMERICAL EXPERIMENT}

We tackle the problem of the robust adaptive lateral control of an autonomous vehicle with unknown tire friction, for a lane-keeping application. We represent the state of a rigid vehicle by its position $\left(p_{x}, p_{y}\right)$, angle $\psi$, velocity $\left(v_{x}, v_{y}\right)$ in the body frame and yaw rate $r$. We denote its mass as $m$, moment of inertia as $I_{z}$, and front and rear axle positions as $a, b$. We consider the Dynamical Bicycle Model described in Chapter 3.2 of [26] parametrised by the unknown front and rear tire friction coefficients $\theta=\left[\begin{array}{ll}C_{\alpha_{f}} & C_{\alpha_{r}}\end{array}\right]^{\top}$, which yields the linear dynamics (1) with

$$
\begin{gathered}
x=\left[\begin{array}{c}
p_{y} \\
\psi \\
v_{y} \\
r
\end{array}\right], \quad A=\left[\begin{array}{cccc}
0 & v_{x} & 1 & 0 \\
0 & 0 & 0 & 1 \\
0 & 0 & 0 & -v_{x} \\
0 & 0 & 0 & 0
\end{array}\right], \quad B=\left[\begin{array}{c}
0 \\
0 \\
\frac{2}{m} \\
\frac{a}{I_{z}}
\end{array}\right], \\
\phi=\frac{-2}{m v_{x} I_{z}}\left[\left[\begin{array}{cccc}
0 & 0 & 0 & 0 \\
0 & 0 & 0 & 0 \\
0 & 0 & I_{z} & a I_{z} \\
0 & 0 & a m & a^{2} m
\end{array}\right],\left[\begin{array}{cccc}
0 & 0 & 0 & 0 \\
0 & 0 & 0 & 0 \\
0 & 0 & I_{z} & -b I_{z} \\
0 & 0 & -b m & b^{2} m
\end{array}\right]\right] .
\end{gathered}
$$

Instead of simply stabilizing the vehicle state $x$, we track the lateral position $y_{r}(t)$ of the lane center. However, we do not have access to a full state reference $x_{r}(t)=$ $\left[y_{r}(t), \psi_{r}(t), v_{y, r}(t), r_{r}(t)\right]^{\top}$ consistent with the dynamics (1). Thus, we define the state $\widetilde{x}=x-\left[y_{r}(t), 0,0,0\right]^{\top}$ and consider the remaining unknown terms $\left[0, \psi_{r}(t), v_{y, r}(t), r_{r}(t)\right]$ and $u_{r}(t)$ as perturbations $\omega(t)$, bounded since $x_{r}, u_{r}$ are assumed to belong to $\mathbb{X}= \pm[3,2,6,6]^{\top}$ and $\mathbb{U}= \pm[10]$. 

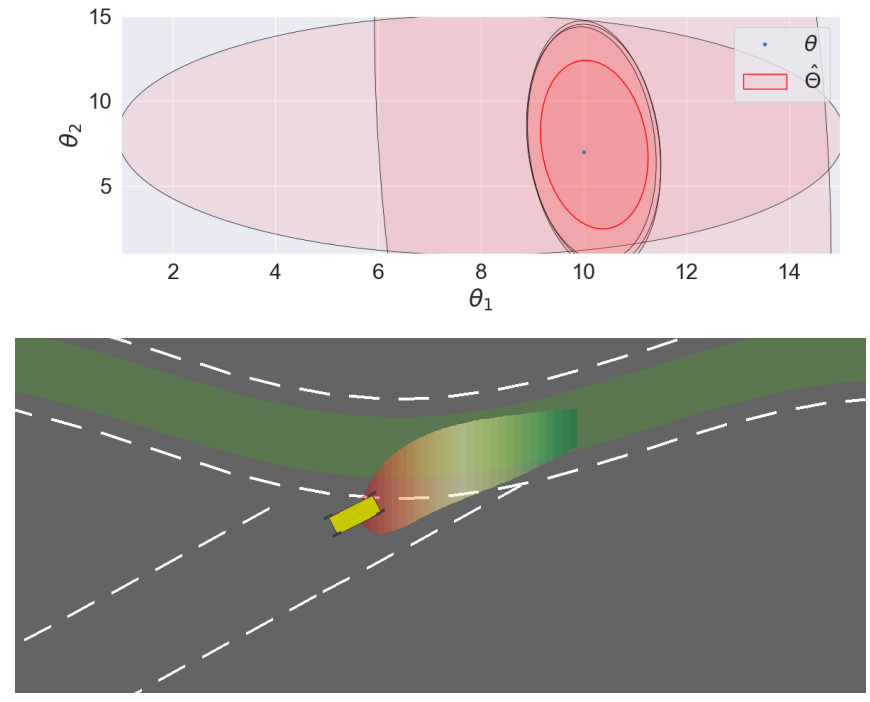

Fig. 2. Top: the model estimation showing the confidence region $\hat{\Theta}(t)$ from (12) at different times $t$. Bottom: a lane keeping application, where a car must follow a lane-center curve under unknown friction and perturbations. $X_{f}$ is shown in green, and $\xi(t)$ as an area with a color gradient.

The Figure 2 depicts our approach. The confidence region $\hat{\Theta}(t)$ from (6) is shown in the top graph, and shrinks with time. To simplify verification of Assumption 4 for this example, an auxiliary preliminary feedback has been applied shifting the eigenvalues of the closed-loop system. The robust stability of this feedback is assessed with the LMI of Theorem 1, and we compute the corresponding basin of attraction $X_{f}$ from [15], represented in green in the bottom subfigure. Then, we use a sampling-based MPC scheme [27] to solve (5) and bring $\xi(t)$ into $X_{f}$ in $T=3 s$. The associated interval prediction $\xi(t)$ from (12) is represented with a color gradient from $t=t_{i}$ (red) to $t=t_{i}+T$ (green). Once the vehicles enters $X_{f}$, we finally switch to the closed-loop feedback (13) following (17) for the rest of the simulation. A video is available at this url.

\section{CONCLUSION}

A robust adaptive MPC algorithm is presented for a partially known linear system subject to disturbances. The peculiarity of the proposed solution consists in utilization of interval predictor. The applicability of the method is demonstrated on a simulated car application.

\section{REFERENCES}

[1] E. Leurent, D. Efimov, T. Raïssi, and W. Perruquetti, "Interval prediction for continuous-time systems with parametric uncertainties," in Proc. IEEE Conference on Decision and Control (CDC), Nice, 2019.

[2] I. Lenz, R. A. Knepper, and A. Saxena, "Deepmpc: Learning deep latent features for model predictive control," in Robotics: Science and Systems, 2015.

[3] V. Mnih, K. Kavukcuoglu, D. Silver, A. A. Rusu, J. Veness, M. G. Bellemare, A. Graves, M. Riedmiller, A. K. Fidjeland, G. Ostrovski, S. Petersen, C. Beattie, A. Sadik, I. Antonoglou, H. King, D. Kumaran, D. Wierstra, S. Legg, and D. Hassabis, "Human-level control through deep reinforcement learning," Nature, vol. 518, no. 7540, pp. 529-533, Feb. 2015.
[4] T. Basar and P. Bernhard, $H$ infinity - Optimal Control and Related Minimax Design Problems: A Dynamic Game Approach. Birkhäuser, 1996, vol. 41

[5] D. Q. Mayne, J. B. Rawlings, C. V. Rao, and P. O. M. Scokaert, "Constrained model predictive control: stability and optimality," Automatica, vol. 36, pp. 789-814, 2000.

[6] D. Q. Mayne, S. V. Ravokic, R. Findeisen, and F. Allgower, "Robust output feedback model predictive control of constrained linear systems: time-varying case," Automatica, vol. 45, pp. 2082-2087, 2009.

[7] S. Dean, H. Mania, N. Matni, B. Recht, and S. Tu, "Regret bounds for robust adaptive control of the linear quadratic regulator," in Advances in Neural Information Processing Systems 31, S. Bengio, H. Wallach, H. Larochelle, K. Grauman, N. Cesa-Bianchi, and R. Garnett, Eds. Curran Associates, Inc., 2018, pp. 4188-4197.

[8] H. Fukushima, T.-H. Kim, and T. Sugie, "Adaptive model predictive control for a class of constrained linear systems based on the comparison model," Automatica, vol. 43, no. 2, pp. 301-308, 2007.

[9] V. Adetola, D. DeHaan, and M. Guay, "Adaptive model predictive control for constrained nonlinear systems," Systems \& Control Letters, vol. 58, no. 5, pp. 320-326, 2009.

[10] V. Adetola and M. Guay, "Robust adaptive mpc for constrained uncertain nonlinear systems," International Journal of Adaptive Control and Signal Processing, vol. 25, no. 2, pp. 155-167, 2011.

[11] A. Aswani, H. Gonzalez, S. S. Sastry, and C. Tomlin, "Provably safe and robust learning-based model predictive control," Automatica, vol. 49, no. 5, pp. 1216-1226, 2013.

[12] B. A. H. Vicente and P. A. Trodden, "Stabilizing predictive control with persistence of excitation for constrained linear systems," Systems \& Control Letters, vol. 126, pp. 58-66, 2019.

[13] D. Efimov, L. Fridman, T. Raïssi, A. Zolghadri, and R. Seydou, "Interval estimation for LPV systems applying high order sliding mode techniques," Automatica, vol. 48, pp. 2365-2371, 2012.

[14] G. Besançon, Nonlinear observers and applications, ser. Lecture notes in control and information. Springer, 2007, vol. 363.

[15] M. Reichhartinger, D. Efimov, and L. Fridman, "Special issue on differentiators," International Journal of Control, vol. 91, no. 9, pp. 1980-1982, 2018.

[16] A. Morgan and K. Narendra, "On the uniform asymptotic stability of certain linear nonautonomous differential equations," SIAM J. Control and Optimization, vol. 15, no. 1, pp. 5-24, 1977.

[17] T. N. Dinh, F. Mazenc, and S.-I. Niculescu, "Interval observer composed of observers for nonlinear systems," European Control Conference, 2014.

[18] V. T. H. Le, "Robust predictive control by zonotopic set-membership estimation." Theses, Supélec, Oct. 2012.

[19] V. Delos and D. Teissandier, "Minkowski Sum of Polytopes Defined by Their Vertices," Journal of Applied Mathematics and Physics (JAMP), vol. 3, no. 1, pp. 62-67, Jan. 2015.

[20] D. Efimov, T. Raïssi, S. Chebotarev, and A. Zolghadri, "Interval state observer for nonlinear time varying systems," Automatica, vol. 49, no. 1, pp. 200-205, 2013.

[21] D. Efimov, T. Raissi, and A. Zolghadri, "Control of nonlinear and LPV systems: Interval observer-based framework," IEEE Transactions on Automatic Control, vol. 58, no. 3, pp. 773-778, 2013.

[22] L. Farina and S. Rinaldi, Positive Linear Systems: Theory and Applications. John Wiley \& Sons, 2000.

[23] E. D. Sontag, "The ISS philosophy as a unifying framework for stabilitylike behavior," in Nonlinear control in the year 2000, Vol. 2 (Paris), ser. Lecture Notes in Control and Inform. Sci. London: Springer, 2001, vol. 259, pp. 443-467.

[24] S. Dashkovskiy, D. Efimov, and E. Sontag, "Input to state stability and allied system properties," Automation and Remote Control, vol. 72, no. 8, pp. 1579-1614, 2011.

[25] H. Michalska and D. Q. Mayne, "Robust receding horizon control of constrained nonlinear systems," IEEE Transactions on Automatic Control, vol. 38, no. 11, pp. 1623-1633, 1993.

[26] M. Awan, Compensation of Low Performance Steering System Using Torque Vectoring. Cranfield University, 2014.

[27] T. H. de Mello and G. Bayraksan, "Monte carlo sampling-based methods for stochastic optimization," Surveys in Operations Research and Management Science, vol. 19, no. 1, pp. 56 - 85, 2014. 